\title{
IgG Endopeptidase in Highly Sensitized Patients Undergoing Transplantation
}

\author{
S.C. Jordan, T. Lorant, J. Choi, C. Kjellman, L. Winstedt, M. Bengtsson, X. Zhang, \\ T. Eich, M. Toyoda, B.-M. Eriksson, S. Ge, A. Peng, S. Järnum, K.J. Wood, \\ T. Lundgren, L. Wennberg, L. Bäckman, E. Larsson, R. Villicana, J. Kahwaji, \\ S. Louie, A. Kang, M. Haas, C. Nast, A. Vo, and G. Tufveson
}

The authors' full names, academic degrees, and affiliations are listed in the Appendix. Address reprint requests to Dr. Jordan at the Comprehensive Transplant Center, Cedars-Sinai Medical Center, 8900 Beverly Blvd., Los Angeles, CA 90048, or at sjordan@cshs.org.

Drs. Jordan and Lorant, and Drs. Vo and Tufveson, contributed equally to this article.

This article was last updated on October 6 , 2017, at NEJM.org.

N EngIJ Med 2017;377:442-53. DOI: 10.1056/NEJMoal612567 Copyright @ 2017 Massachusetts Medical Society.
ABSTRACT

\section{BACKGROUND}

Donor-specific antibodies create an immunologic barrier to transplantation. Current therapies to modify donor-specific antibodies are limited and ineffective in the most highly HLA-sensitized patients. The IgG-degrading enzyme derived from Streptococcus pyogenes (IdeS), an endopeptidase, cleaves human IgG into $\mathrm{F}\left(\mathrm{ab}^{\prime}\right)_{2}$ and Fc fragments inhibiting complement-dependent cytotoxicity and antibody-dependent cellular cytotoxicity, which suggests that IdeS might be useful for desensitization. We report on the combined experience of two independently performed open-label, phase 1-2 trials (conducted in Sweden and the United States) that assessed the efficacy of IdeS with regard to desensitization and transplantation of a kidney from an HLA-incompatible donor.

\section{METHODS}

We administered IdeS to 25 highly HLA-sensitized patients (11 patients in Uppsala or Stockholm, Sweden, and 14 in Los Angeles) before the transplantation of a kidney from an HLA-incompatible donor. Frequent monitoring for adverse events, outcomes, donor-specific antibodies, and renal function was performed, as were renal biopsies. Immunosuppression after transplantation consisted of tacrolimus, mycophenolate mofetil, and glucocorticoids. Patients in the U.S. study also received intravenous immune globulin and rituximab after transplantation to prevent antibody rebound.

\section{RESULTS}

Recipients in the U.S. study had a significantly longer cold ischemia time (the time elapsed between procurement of the organ and transplantation), a significantly higher rate of delayed graft function, and significantly higher levels of class I donorspecific antibodies than those in the Swedish study. A total of 38 serious adverse events occurred in 15 patients ( 5 events were adjudicated as being possibly related to IdeS). At transplantation, total IgG and HLA antibodies were eliminated. A total of 24 of 25 patients had perfusion of allografts after transplantation. Antibodymediated rejection occurred in 10 patients ( 7 patients in the U.S. study and 3 in the Swedish study) at 2 weeks to 5 months after transplantation; all these patients had a response to treatment. One graft loss, mediated by non-HLA IgM and IgA antibodies, occurred.

\section{CONCLUSIONS}

IdeS reduced or eliminated donor-specific antibodies and permitted HLA-incompatible transplantation in 24 of 25 patients. (Funded by Hansa Medical; ClinicalTrials .gov numbers, NCT02224820, NCT02426684, and NCT02475551.) 
LTHOUGH RENAL TRANSPLANTATION IS considered to be the treatment of choice for patients with end-stage renal disease, ${ }^{1,2}$ rates of transplantation remain low among patients with high levels of preformed anti-HLA antibodies. In such cases, the immunologic barrier, which is linked to an increased risk of antibody-mediated rejection and poor graft survival, ${ }^{3}$ remains a major deterrent to transplantation. Approximately $30 \%$ of the patients on transplantation waiting lists currently have evidence of sensitization in the form of alloantibodies that were generated because of exposure to previous transplants, blood transfusions, pregnancy, or other events. The presence of a panel-reactive antibody level of at least $80 \%$ to the loci tested for matching (i.e., a high level of sensitization) creates difficulty in finding matched kidneys from compatible donors and results in approximately one of seven such patients receiving a kidney transplant in a given year. ${ }^{4}$

Various attempts that have been designed to improve transplantation rates among highly sensitized patients incorporate the use of desensitization protocols combining B-lymphocyte-depleting agents (e.g., rituximab and anti-CD20), ${ }^{5}$ intravenous immune globulin, and plasmapheresis, ${ }^{5-8}$ combined with better stratification of immunologic risk with the use of sensitive donor-specific HLA-antibody screening and avoidance techniques. Recent data support the lifesaving benefits of desensitization and transplantation, as compared with remaining on dialysis. ${ }^{9,10}$ However, current desensitization protocols often result in incomplete removal of donor-specific antibodies, rebound antibody production, and an increased risk of acute and chronic antibody-mediated rejection, which are the primary causes of early graft loss and return to dialysis, with the attendant emotional consequences for the patients and financial consequences for the health care system. ${ }^{11-13}$ Currently, there are no approved drugs for desensitization and the management of donor-specific antibody-induced antibody-mediated rejection. ${ }^{14}$ On the basis of these observations, there is a clinical need for new therapies to improve the process and outcomes of desensitization.

The IgG-degrading enzyme derived from Streptococcus pyogenes (IdeS; GenBank accession number, ADF13949.1) is a recombinant cysteine protease of $S$. pyogenes produced in Escherichia coli that cleaves all four human subclasses of IgG with strict specificity. IgG-degrading activity is a common strategy that is used by pathogenic bacteria. IdeS hydrolyzes human IgG at Gly236 in the lower hinge region of the IgG heavy chains. ${ }^{15,16}$ That activity is important, since the Fc region of IgG is critical for interaction with Fc receptors and complement binding. Thus, the proteolytic activity on IgG molecules at this site prevents the occurrence of IgG-mediated antibody-dependent cellular cytotoxicity and complement-mediated cytotoxicity, two processes that are critical for antibody rejection (Fig. 1A). Here we report on the use of Ides as a therapy to disable pathogenic IgG donor-specific antibodies as a desensitization strategy in highly sensitized candidates for transplantation.

METHODS

\section{PATIENTS AND STUDY DESIGN}

We combined data from two separate open-label, single-group, phase 1-2 studies investigating the safety and efficacy of Ides to reduce or remove pathogenic donor-specific antibodies in order to allow HLA-incompatible kidney transplantation. The studies were performed independently at Cedars-Sinai Medical Center, Los Angeles, and at Uppsala University, Uppsala, Sweden, and Karolinska Institutet, Stockholm. The study protocols (available with the full text of this article at NEJM.org) were approved at each site by an institutional review board or ethics committee. All the patients provided written informed consent.

An initial dose-finding study conducted in Sweden examined the efficacy of IdeS to remove HLA antibodies in highly HLA-sensitized patients. Two subsequent studies, one conducted in the United States and one conducted in Sweden, examined the effectiveness and safety profile of the streptococcal IgG endopeptidase IdeS given before kidney transplantation in highly sensitized patients to reduce or eliminate donor-specific antibodies and to allow transplantation from an incompatible donor without early antibody rejection.

Eligible patients were 18 to 70 years of age, were undergoing dialysis for end-stage renal disease, and were awaiting kidney transplantation on either the United Network for Organ Sharing waiting list (in the United States) or the Scandiatransplant waiting list (in Sweden). All the patients had extensive sensitization, with a median calculated panel-reactive antibody level of 95\% (range, 


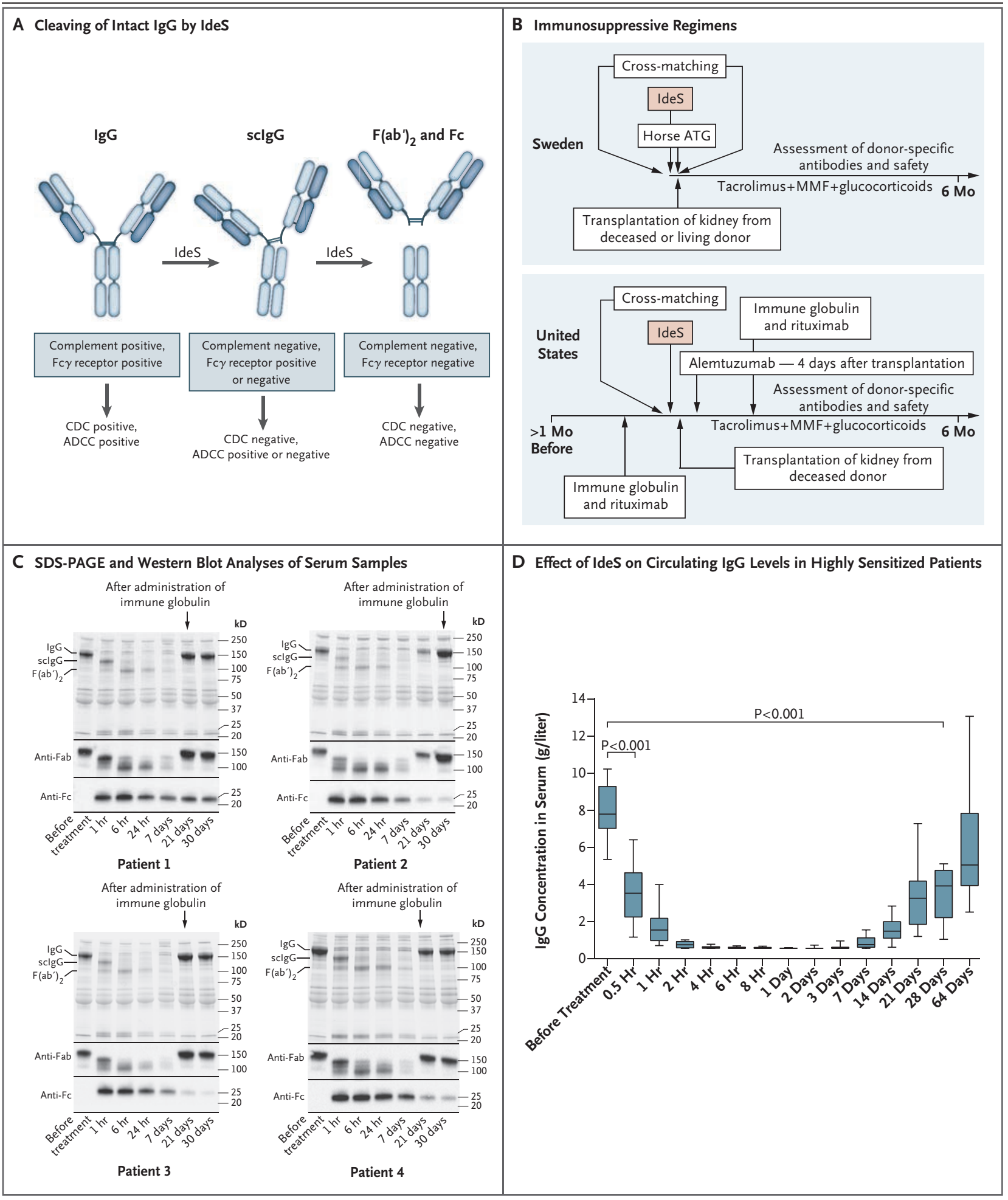

The New England Journal of Medicine

Downloaded from nejm.org at the Bodleian Libraries of the University of Oxford on October 15, 2018. For personal use only. No other uses without permission. Copyright (C) 2017 Massachusetts Medical Society. All rights reserved. 
Figure 1 (facing page). IdeS Analysis and Mechanism. Panel A shows how the IgG-degrading enzyme derived from Streptococcus pyogenes (IdeS) cleaves intact IgG. Intact human IgG, regardless of isotype, is cleaved by IdeS in two steps. Step one results in a single cleavage of the IgG molecule (sclgG) in which one heavy chain remains intact. Step two generates a fully cleaved product that cannot mediate complement-dependent cytotoxicity (CDC) or antibody-dependent cell-mediated cytotoxicity (ADCC) by means of Fc $\gamma$ receptors. Panel B shows a flow diagram of the immunosuppressive regimens used before and after transplantation in the studies conducted in Sweden and the United States. Immune globulin was administered intravenously. A total of 12 of 14 patients in the U.S. study received desensitization with immune globulin and rituximab before transplantation. All the patients received these agents after transplantation to prevent antibody rebound. ATG denotes antithymocyte globulin, and MMF mycophenolate mofetil. Panel $\mathrm{C}$ shows the results on serum samples obtained from 4 patients treated with IdeS. Samples were obtained before and at the indicated time points after treatment and were subjected to sodium dodecyl sulfate-polyacrylamide-gel electrophoresis (SDS-PAGE) (top), Western blot with Fab-specific reagent (middle), and Western blot with Fc-specific reagent (bottom). The data show that at 1 hour, IgG is cleaved into sclgG and $F\left(a b^{\prime}\right)_{2}$ and $F c$, with no intact IgG remaining. At 6 hours, IgG is completely cleaved into $F\left(a b^{\prime}\right)_{2}$ and Fc. The box plot in Panel D shows the total serum IgG levels before and at consecutive time points after treatment (in 10 patients). The horizontal line in the boxes shows the median, the top and bottom of the boxes the interquartile range, and the I bars the range. $P$ values were calculated by a repeated-measures oneway analysis of variance with Dunn's multiple-comparison test.

22 to 100), and had a clinically significant sensitization history. The selection of the patients was based on levels of sensitization and wait time that had been so long as to include frequent offers of transplants obtained from deceased donors that had resulted in positive cross-matches and donor-specific antibodies that had previously prohibited transplantation.

The acceptance criteria for HLA-incompatible organs in recipients in the United States have been reported previously. Briefly, these criteria include a negative complement-cytotoxicity cross-match, a negative flow-cytometric cross-match, or a positive T-cell and B-cell flow-cytometric cross-match with approximately 250 channel shifts or less and usually donor-specific antibody positivity. ${ }^{13,17}$
(Channel shift refers to a method of measuring the intensity of light signals generated from dyespecific fluorescent-labeled antibodies binding to HLA antigens on target cells. Light signals are converted by an analogue-to-digital converter so signals can be processed by a binary computer. The intensity of the binding is measured as degrees of channel shifts relative to negative controls.) In Sweden, patients were eligible if they had had at least two anti-HLA antibodies with a mean fluorescence intensity of 3000 or more. Eight patients participated in the initial dose-finding study, which did not include transplantation and which was used to establish doses of IdeS to be used in the transplantation trials. A total of 25 patients (14 patients in the United States and 11 in Sweden) met the criteria outlined above and participated in the transplantation studies.

Donor-specific antibodies were detected with the use of solid-phase assay systems that were currently in use at the HLA laboratory of each hospital. ${ }^{17}$ After safety assessments, the patients proceeded to receive a transplant from an incompatible donor. Study patients who received a kidney transplant had samples obtained before IdeS treatment for the assessment of donor-specific antibodies and flow-cytometric cross-matching and also underwent studies after treatment that included monitoring for efficacy of IgG cleavage at prespecified time points.

Patients who underwent transplantation received IdeS at a dose of $0.24 \mathrm{mg}$ per kilogram of body weight (in the United States) or at a dose of $0.25 \mathrm{mg}$ per kilogram or $0.50 \mathrm{mg}$ per kilogram (in Sweden; both doses were investigated in a dose-finding study). IdeS was administered intravenously on day 0 , usually 4 to 6 hours before transplantation. Cross-match and donor-specificantibody tests were conducted at 6 hours and 24 hours and on days 7, 30, 60, 90 (only in the U.S. study), and 180 (only in the U.S. study) after treatment to determine the efficacy of IdeS. Patients in the Swedish cohort received induction with horse antithymocyte globulin (Atgam, Pfizer) for 4 days after transplantation. Horse antithymocyte globulin is not susceptible to digestion by Ides (unpublished data). Patients in the U.S. cohort received induction with alemtuzumab, at a dose of $30 \mathrm{mg}$ administered subcutaneously 4 days after transplantation. Patients in the U.S. and Swedish 
studies continued to receive standard immunosuppression, as reported previously. ${ }^{5}$ The levels of tacrolimus in blood were maintained at 10 to $12 \mathrm{ng}$ per milliliter in the Swedish study and at 8 to $10 \mathrm{ng}$ per milliliter in the U.S. study.

All the patients received prophylactic antibiotic agents to prevent bacterial infection in the absence of IgG antibodies. Patients in the U.S. study received intravenous immune globulin at a dose of $2 \mathrm{~g}$ per kilogram (maximum dose, $140 \mathrm{~g}$ ) on days 7 to 14 after transplantation. Patients in the U.S. study who did not receive rituximab before kidney transplantation received rituximab at a dose of $375 \mathrm{mg}$ per square meter of body-surface area on days 14 to 21 after transplantation. A summary of the treatment protocols is shown in Figure 1B. Humanized monoclonal antibodies and intravenous immune globulin were administered beyond the half-life of IdeS in order to avoid digestion.

Renal-allograft biopsies were performed to assess for antibody-mediated rejection when allograft dysfunction was noted. In addition, perprotocol biopsies were performed at 6 months in the two studies. Assessments in the two studies included C4d staining with the use of the Banff 2013 criteria. ${ }^{18}$ A condensed version of the pathological criteria for the assessment of antibodymediated rejection according to the Banff 2013 criteria is shown in Figure S1 in the Supplementary Appendix, available at NEJM.org.

\section{CLINICAL ASSESSMENT}

Assessments included routine laboratory tests, measurement of panel-reactive antibodies and donor-specific antibodies, assessment of vital signs, and the collection of data related to adverse events and serious adverse events. All the adverse events and serious adverse events were recorded, graded, and reported to the institutional review board at each center, to the study sponsor (Hansa Medical), and to regulatory authorities. Samples for the analysis of IdeS levels and qualitative analysis of patients' IgG levels were assessed with the use of sodium dodecyl sulfate-polyacrylamide-gel electrophoresis (SDS-PAGE). ${ }^{19}$ IdeS cleavage and clearance of the $\mathrm{Fc}$ and $\mathrm{F}\left(\mathrm{ab}^{\prime}\right)_{2}$ fragments were analyzed with the use of enzyme-linked immunosorbent assay (ELISA) methods, as previously described. ${ }^{17}$ The SDS-PAGE and Western blot analy- ses were performed according to the manufacturers' instructions under nonreduced conditions. ${ }^{19}$

\section{STUDY OVERSIGHT}

The preliminary dose-finding study was conducted in Sweden. The two transplantation studies had a phase 1-2 single-group design. The U.S. study was approved by the institutional review board at Cedars-Sinai Medical Center. The Swedish study was approved by the Uppsala regional ethics committee and the Swedish Medical Product Agency. The studies were conducted in accordance with the Declaration of Helsinki, with the ethics guidelines based on federal regulations and the Common Rule. Cedars-Sinai Medical Center also has a federal-wide assurance. The U.S. study was an investigator-initiated study (by the first author). The Swedish study was initiated by Hansa Medical. The studies were designed, conducted, and evaluated solely by the investigators after approval and funding by Hansa Medical after confidentiality agreements were in place. The data were gathered and analyzed and the manuscript was prepared by the investigators, all of whom vouch for the completeness and accuracy of the results and for the fidelity of the studies to the protocols.

\section{STATISTICAL ANALYSIS}

Statistical analysis of the characteristics of the patients and donors was performed with the use of a Mann-Whitney U test. The total serum IgG levels were compared with the use of a repeatedmeasures one-way analysis of variance with Dunn's multiple-comparison test. The highest levels of donor-specific antibodies were compared between the studies with the use of a repeated-measures one-way analysis of variance and Sidak's test for multiple comparisons. A P value of less than 0.05 was considered to indicate statistical significance.

\section{RESULTS}

\section{CHARACTERISTICS OF THE PATIENTS}

Eight patients with end-stage renal disease participated in the dose-finding study. An additional 25 highly sensitized patients who were undergoing dialysis and awaiting transplantation of a kidney from a deceased or living donor were enrolled in the two transplantation studies. The median 


\begin{tabular}{|c|c|c|c|c|}
\hline Characteristic & $\begin{array}{c}\text { Total } \\
(N=25)\end{array}$ & $\begin{array}{l}\text { Sweden } \\
(N=11)\end{array}$ & $\begin{array}{l}\text { United States } \\
\qquad(\mathrm{N}=14)\end{array}$ & P Value \\
\hline \multicolumn{5}{|l|}{ Recipient } \\
\hline Age $-y r$ & $46.2 \pm 14$ & $52.4 \pm 12.3$ & $41.4 \pm 13.9$ & 0.05 \\
\hline Male sex - no. (\%) & $11(44)$ & $4(36)$ & $7(50)$ & 0.69 \\
\hline At least 1 previous kidney transplant received — no. (\%) & $14(56)$ & $5(45)$ & $9(64)$ & 0.27 \\
\hline \multicolumn{5}{|l|}{ Donor } \\
\hline Deceased — no. (\%) & $23(92)$ & $9(82)$ & $14(100)$ & 0.18 \\
\hline Cold ischemia time - $\mathrm{hr}$ & $15.8 \pm 7.5$ & $10.6 \pm 6.8$ & $19.9 \pm 5.2$ & $<0.001$ \\
\hline Delayed graft function — no./total no. (\%) & $10 / 24(42)$ & $0 / 11$ & $10 / 13(77)$ & $<0.001$ \\
\hline \multicolumn{5}{|l|}{ Immunologic variables } \\
\hline Anti-HLA donor-specific antibody positive — no. (\%) & $22(88)$ & $9(82)$ & $13(93)$ & 0.18 \\
\hline No. of anti-HLA donor-specific antibodies & $2.3 \pm 1.8$ & $2.2 \pm 1.6$ & $2.4 \pm 1.9$ & 0.79 \\
\hline \multicolumn{5}{|l|}{ Mean fluorescence intensity } \\
\hline Class 1 & $5660 \pm 2364$ & $4192 \pm 2372$ & $6375 \pm 1996$ & 0.04 \\
\hline Class II & $8199 \pm 5639$ & $10,464 \pm 7051$ & $6500 \pm 3571$ & 0.06 \\
\hline $\begin{array}{l}\text { Negative anti-HLA donor-specific antibodies at } 1 \text { to } 6 \mathrm{hr} \\
\text { after treatment - no. (\%) }\end{array}$ & $25(100)$ & $11(100)$ & $14(100)$ & $>0.99$ \\
\hline Positive cross-match at transplantation - no. (\%) $†$ & $20(80)$ & $7(64)$ & $13(93)$ & 0.13 \\
\hline $\begin{array}{l}\text { Estimated GFR at } 1 \text { to } 6 \mathrm{mo} \text { after transplantation } \\
\qquad-\mathrm{ml} / \mathrm{min} / 1.73 \mathrm{~m}^{2}\end{array}$ & $58 \pm 30$ & $49 \pm 13$ & $70 \pm 36$ & 0.14 \\
\hline Follow-up - mo & $4.7 \pm 1.9$ & $5.7 \pm 0.9$ & $4.0 \pm 2.4$ & 0.03 \\
\hline Graft loss - no. (\%) & $1(4)$ & 0 & $1(7)$ & $>0.99$ \\
\hline
\end{tabular}

* Plus-minus values are means \pm SD. Data on the number of anti-HLA donor-specific antibodies were missing for two patients in the Swedish study, data on the mean fluorescence intensity for five patients in the Swedish study and for four in the U.S. study, and data on the estimated glomerular filtration rate (GFR) and duration of follow-up for one patient in the U.S. study. Statistical analysis was performed with the use of a Mann-Whitney $U$ test. A P value of less than 0.05 was considered to indicate statistical significance.

$\uparrow$ Positive cross-match was defined as a T-cell flow-cytometric result that was greater than 50 mean channel shifts, a T-cell pronase result that was greater than 70 mean channel shifts, a B-cell flow-cytometric result that was greater than 100 mean channel shifts, and a B-cell pronase result that was greater than 130 mean channel shifts. Cross-match positivity was not a requirement for inclusion in the Swedish trial, whereas the U.S. trial required donor-specific antibody positivity or cross-match positivity (or both).

calculated panel-reactive antibody level was $96 \%$ (range, 82 to 100) in the patients in the U.S. study and $81 \%$ (range, 22 to 100) in the patients in the Swedish study. Patients in the U.S. study had a significantly longer cold ischemia time (the time elapsed between procurement of the organ and transplantation), a significantly higher rate of delayed graft function, and a significantly higher mean fluorescence intensity for HLA class I antibodies at the time of transplantation than did those in the Swedish study. A total of 22 of 25 patients had donor-specific antibodies present at the time of transplantation. No patient had detectable HLA antibodies or donor-specific anti- bodies immediately after transplantation. The characteristics of the patients and donors and the immunologic characteristics of the patients are shown in Table 1.

\section{IDES AND SERUM IGG LEVELS}

Patients were admitted to the hospital before transplantation and received IdeS over a period of 15 minutes approximately 4 to 6 hours before the receipt of a kidney transplant from an incompatible donor. Laboratory values were obtained before transplantation and at specified times after transplantation. A total of 24 of 25 patients had perfusion of allografts after transplantation. 


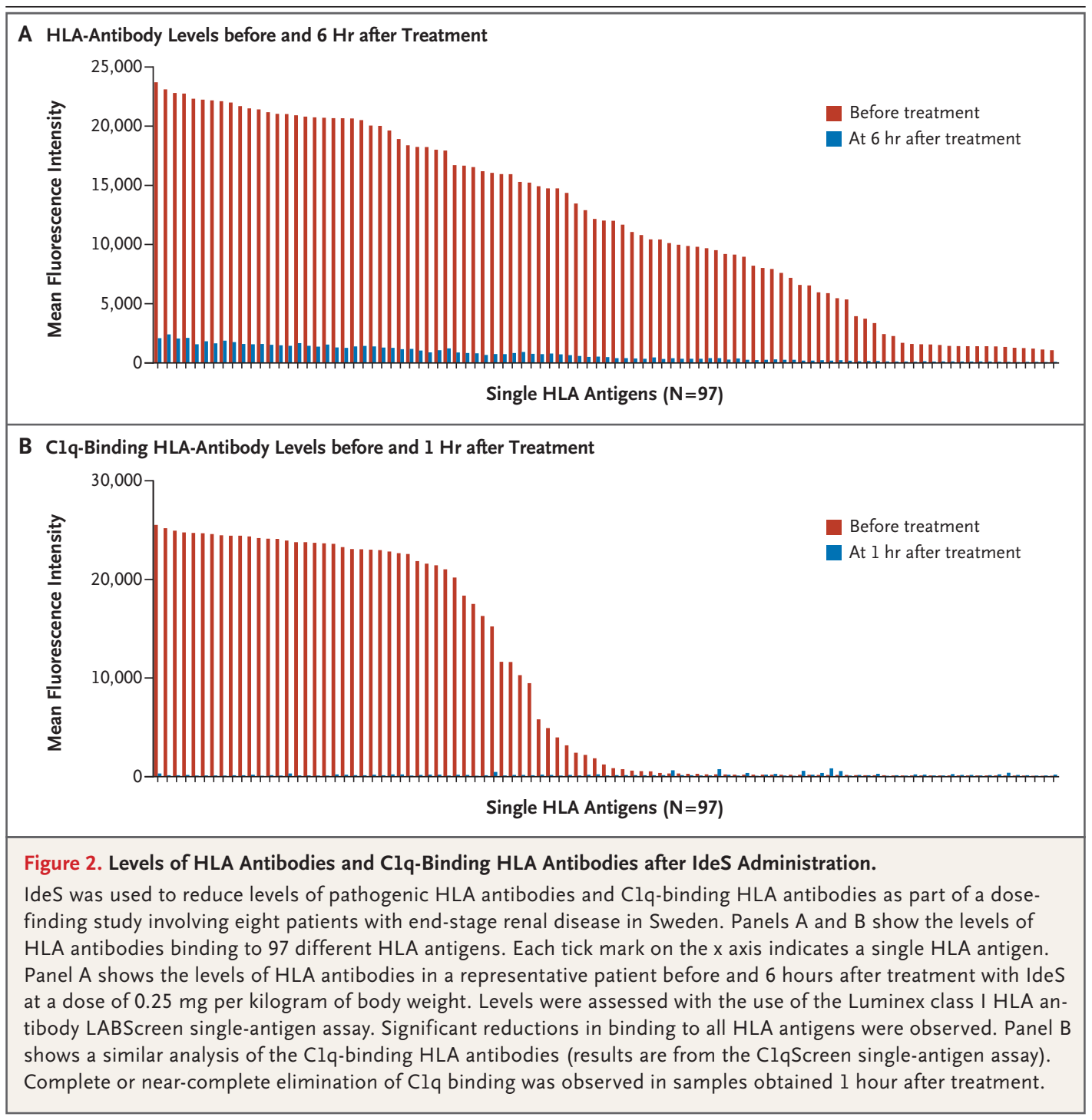

Subsequent assessments of SDS-PAGE, total IgG, and Western blot analyses of patients' serum specimens obtained before and after treatment with IdeS were performed. SDS-PAGE analysis of serum specimens revealed reductions in the total IgG level beginning after the infusion of IdeS (Fig. 1C). By 6 hours after the start of the infusion, all the IgG molecules are completely cleaved into $\mathrm{Fc}$ and $\mathrm{F}\left(\mathrm{ab}^{\prime}\right)_{2}$ fragments, which probably reduces their pathogenicity. ${ }^{19}$ All the IgG molecules are inactivated for approximately 1 to 2 weeks, when new IgG synthesis is detected. ${ }^{19}$ The large bands shown in the IgG region at 21 to 30 days occurred after the receipt of intravenous infu- sions of immune globulin. Western blot analysis confirmed the complete cleavage of IgG molecules into $\mathrm{Fc}$ and $\mathrm{F}\left(\mathrm{ab}^{\prime}\right)_{2}$ fragments. Figure $\mathrm{S} 2$ in the Supplementary Appendix shows the dynamics of total IgG, $\mathrm{Fc}$, and $\mathrm{F}\left(\mathrm{ab}^{\prime}\right)_{2}$ fragments in the serum specimens obtained from 10 IdeS-treated patients, as measured with the use of ELISA techniques. Intact IgG levels begin to decline rapidly; little intact IgG was present at 6 hours after treatment with IdeS. No intact IgG was seen at or after 7 days. Figure 1D shows the serum IgG levels after treatment with IdeS in 10 patients in the Swedish study. There was a significant reduction in the total IgG level that persisted for 28 days. 


\section{HLA-ANTIBODY AND DONOR-SPECIFIC-ANTIBODY} LEVELS

Complement-activating donor-specific antibodies are known to severely injure and rapidly destroy the allograft. ${ }^{20}$ Thus, IdeS would need to inactivate pathogenic donor-specific antibodies, specifically those that can activate complement, if it will work as a potential desensitization agent. The dosefinding study involving eight HLA-sensitized patients who were undergoing dialysis evaluated the ability of IdeS to reduce the levels of C1q-binding and total HLA antibodies. Data from a representative patient with multiple, strong C1q-binding HLA antibodies are shown in Figure 2A and 2B. Briefly, near-complete inhibition of C1q-binding HLA antibodies was seen 1 hour after treatment. The levels of all HLA antibodies were significantly reduced at 6 hours after treatment.

We also examined sequential lymphocyte counts before and after treatment with IdeS in 8 patients in the Swedish study who underwent transplantation and received induction with horse antithymocyte globulin (Fig. S3 in the Supplementary Appendix). The course of lymphocyte depletion and repletion was similar to that observed in 36 patients in the United States who underwent transplantation after desensitization without IdeS. ${ }^{21}$

We assessed the level of circulating HLA antibodies and donor-specific antibodies at multiple time points before treatment with IdeS and after transplantation. All the patients had near-complete or complete reductions of levels of HLA antibodies and donor-specific antibodies at 6 hours and 24 hours after treatment. Data are presented separately for the two studies. The mean donorspecific-antibody levels in the two studies before treatment and at 6 hours and 1 month after treatment are shown in Figure 3A. Despite similar levels of donor-specific antibodies before transplantation in the U.S. and Swedish studies, significant reductions in the sum of donor-specific antibodies and the highest levels of donor-specific antibodies were seen in the U.S. cohort at 1 month after transplantation (Fig. 3A). Figure S4A in the Supplementary Appendix shows representative data from three patients in the Swedish study. Levels of HLA antibodies and donor-specific antibodies remained undetectable until 7 to 14 days after transplantation, when rebound occurred. In contrast, the U.S. cohort had fewer patients with rebound and lower levels of HLA antibodies after treatment with IdeS (Fig. S4B in the Supplementary Appendix). Thus, in all, significant differences were seen between the groups of patients in the U.S. study and the Swedish study, indicating that the anamnestic responses were blunted in the patients in the U.S. study at 1 month, which probably reflects the use of intravenous immune globulin plus rituximab before and after transplantation. ${ }^{22,23}$

\section{CLINICAL OUTCOMES}

Three patients in the Swedish study had antibodymediated rejection at a mean of 2 weeks after transplantation that was associated with rebound of donor-specific antibodies and C4d+ on biopsies. Per-protocol biopsies that were performed at 6 months revealed minimal inflammation in 9 of 11 patients. Seven patients in the U.S. study had inflammation on renal biopsy that was detected at a mean of 3.6 months after transplantation. Two patients in the U.S. study met the strict Banff 2013 criteria for antibody-mediated rejection (detected at 5 months and at 2 months after IdeS treatment); the rejection was associated with a rise in the levels of donor-specific antibodies and resolved after treatment. Renal function was generally good in the two groups after transplantation (Fig. 3B). The pathological features on biopsy specimens obtained from patients with antibody-mediated rejection and on the specimens obtained in per-protocol biopsies conducted at 6 months in the two studies are shown in Figure 3C. The patients with antibody rejection in the Swedish study had higher C4d+ scores than did those in the U.S. study, whereas low levels of inflammation were seen in the per-protocol biopsies that were conducted in the two groups at 6 months after transplantation.

One patient in the U.S. study had hyperacute rejection immediately after revascularization. This event was unexpected, since the tests for crossmatches and donor-specific antibodies were negative after IdeS treatment and before transplantation. Extensive investigations after the rejection showed high-titer IgM and IgA antibody reactive with donor-allograft endothelium, which we speculate was probably responsible for the immediate graft loss. Subsequent assessments showed no evidence of IgM anti-HLA or donor-specific anti- 


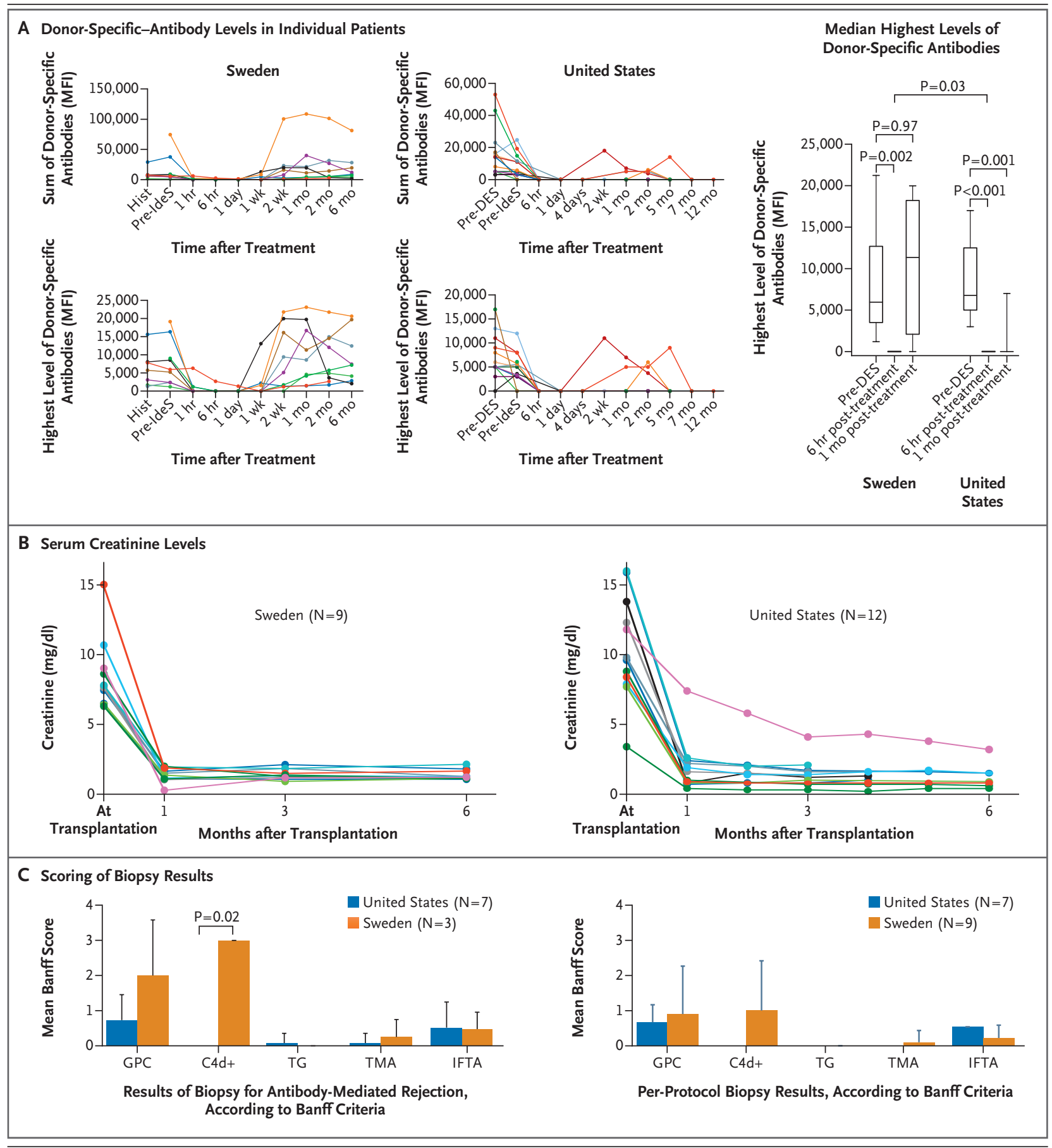

bodies. Thus, the antibody appears to be a non- concern. Data from healthy volunteers in a phase HLA antibody that cannot be cleaved by IdeS. 1 study $^{19}$ showed no signal for an increased in-

\section{ADVERSE EVENTS}

The possibility of serious infectious complications related to IdeS treatment was an anticipated cidence of infection after IdeS infusion. In the U.S. cohort, we observed no significant infectious complications. Patients were monitored monthly for cytomegalovirus, Epstein-Barr virus, polyoma- 
Figure 3 (facing page). Donor-Specific-Antibody Levels, Creatinine Levels, and Scoring of Biopsy Results.

Panel A shows the comparison of the sum of all donor-specific antibodies (top) and the highest levels of donor-specific antibodies (bottom) among 9 patients treated in the Swedish study and 14 patients treated in the U.S. study. Levels were assessed according to the mean fluorescence intensity (MFI). Hist denotes historical data, and DES desensitization. Panel A also shows a comparison of the median highest levels of donor-specific antibodies before desensitization and at 6 hours and 1 month after IdeS treatment; the horizontal line in the boxes shows the median, the top and bottom of the boxes the interquartile range, and the I bars the range. The data show a significant difference in donor-specific-antibody rebound between the studies; results are from a repeated-measures one-way analysis of variance and Sidak's test for multiple comparisons. Panel B shows the course of serum creatinine levels before and after transplantation in patients in the two studies. Overall, the renal function was good, although 1 patient in the U.S. study had prolonged delayed graft function that was due to suboptimal quality of the donated kidney. To convert creatinine values to micromoles per liter, multiply by 88.4. Panel $C$ shows the pathological features of biopsies performed because of antibody-mediated rejection in 7 patients in the U.S. study and in 3 in the Swedish study in whom antibody-mediated rejection developed after transplantation (left). Also shown are the pathological features of per-protocol biopsies performed at 6 months after receipt of IdeS in 7 patients in the U.S. study and in 9 patients in the Swedish study (right). Scoring of various values (complement factor $4 \mathrm{~d}$ deposition [C4d+], interstitial fibrosis and tubular atrophy [IFTA], glomerulitis plus peritubular capillaritis [GPC], transplant glomerulopathy [TG], and thrombotic microangiopathy [TMA]) was based on Banff 2013 criteria. ${ }^{18}$ T bars indicate I SD. Significantly less C4d deposition was seen in the U.S. cohort than in the Swedish cohort. Panel C also shows the results of the per-protocol biopsies (right). Low levels of immune injury were seen in the two cohorts.

virus type $\mathrm{BK}$, and JC viremia, with no patients having positivity.

A complete summary of serious adverse events is shown in Table 2. No deaths occurred in this study. A total of 38 serious adverse events were observed in 15 patients; 5 of these events were considered by the investigators as being possibly attributable to IdeS. There were 13 infectious complications that generally responded to treatment. However, in the Swedish study, 1 patient had prolonged parvovirus B19 viremia and 1 had persistent myalgias after the IdeS infusion.
DISCUSSION

The data in this study show a significant reduction in levels of IgG HLA alloantibodies and donorspecific antibodies after treatment with IdeS. The reduction or elimination of donor-specific antibodies allowed for successful transplantation in 24 of 25 patients. The single allograft loss was due to hyperacute rejection in a patient who appeared to have a non-HLA antibody. Outcomes in the patients treated with IdeS in the two cohorts were good, including similar graft survival and overall survival among patients, renal function, and incidence of antibody-mediated rejection up to 1.5 years after transplantation.

Nearly 50 years ago, Terasaki and Russell and colleagues identified alloantibodies as major immunologic barriers to successful transplantation and graft survival. ${ }^{24,25}$ Despite advancements in desensitization, the efficient removal of pathogenic HLA antibodies is still a considerable medical challenge. ${ }^{20,26}$

In 2002, a cysteine protease, IdeS, was discovered and purified from S. pyogenes. ${ }^{27,28}$ IdeS has a unique specificity for IgG and is a potent virulence factor produced by $S$. pyogenes, because removing the Fc region of host IgG essentially ablates humoral immunity given that cleaved IgG can no longer activate complement or mediate antibody-dependent cellular cytotoxicity. ${ }^{29,30}$ Thus, the inactivation of IgG-mediated effector pathways by IdeS may help to prevent antibody-mediated injury to allografts. IdeS also cleaves B-cell receptors from circulating B cells, with the resultant inhibition of antigenspecific B-cell IgG responses in vitro. ${ }^{29}$ The data presented here support the hypothesis that IdeS may reduce or eliminate donor-specific antibodies, which may facilitate transplantation from an HLA-incompatible donor without the risk of early antibody-mediated rejection. Our data should be interpreted cautiously, since only a small number of highly sensitized patients were included in our study and the risk of rebound of donorspecific antibodies is not addressed by IdeS. The use of intravenous immune globulin and rituximab after transplantation probably prevents rebound donor-specific-antibody responses to some extent. ${ }^{22,23}$

A major strength of this study lies in showing the consistent ability of IdeS to reduce or elimi- 


\begin{tabular}{|c|c|c|}
\hline Event & No. of Events & $\begin{array}{l}\text { Investigators' Assessment of Relatedness } \\
\text { of Event to IdeS Treatment }\end{array}$ \\
\hline \multicolumn{3}{|l|}{ Bacterial infection } \\
\hline Urinary tract infection & 5 & Not related \\
\hline Blood infection & 3 & Possibly related (in 1) and unlikely to be related (in 2) \\
\hline Abdominal infection & 1 & Possibly related \\
\hline Catheter-site infection & 1 & Possibly related \\
\hline Pneumonia & 2 & Unlikely to be related (in 1) and not related (in 1) \\
\hline Viral infection with parvovirus & 1 & Possibly related \\
\hline Lymphocele & 1 & Not related \\
\hline Renal-artery stenosis & 1 & Not related \\
\hline Ureteric obstruction & 2 & Not related \\
\hline Cholelithiasis or cholecystitis & 2 & Unlikely to be related (in 1) and not related (in l) \\
\hline Leukopenia & 1 & Not related \\
\hline Elevated creatinine level & 1 & Not related \\
\hline \multicolumn{3}{|l|}{ Rejection† } \\
\hline Antibody-mediated rejection & 5 & Not related \\
\hline Cell-mediated rejection & 1 & Not related \\
\hline $\begin{array}{l}\text { Both antibody-mediated rejection and } \\
\text { cell-mediated rejection }\end{array}$ & 2 & Not related \\
\hline $\begin{array}{l}\text { Antibody-mediated rejection mediated } \\
\text { by IgM antibody }\end{array}$ & 1 & Not related \\
\hline Abdominal pain & 3 & Not related \\
\hline Anemia & 1 & Not related \\
\hline Fever & 1 & Not related \\
\hline $\begin{array}{c}\text { Atrial fibrillation and exacerbation of } \\
\text { congestive heart failure }\end{array}$ & 1 & Not related \\
\hline Malignant hyperthermia & 1 & Not related \\
\hline Myalgia & 1 & Possibly related \\
\hline
\end{tabular}

* IdeS is an IgG-degrading enzyme derived from Streptococcus pyogenes. All the serious adverse events were resolved (patients recovered) except for the event of myalgia, which is an ongoing serious adverse event.

$\uparrow$ Rejection was counted per patient rather than per event.

nate donor-specific antibodies to a level that allows the transplantation of a kidney from an HLA-incompatible donor. The major limitations of this study include the small number of patients and the slightly different protocols that were used at the involved centers. Larger, multicenter trials are indicated.

In conclusion, the administration of IdeS before transplantation in highly sensitized patients appeared to be effective as a means of reducing or eliminating donor-specific antibodies, a strategy that may improve transplantation rates among patients with HLA incompatibility.
Supported by research grants from Hansa Medical.

Disclosure forms provided by the authors are available with the full text of this article at NEJM.org.

We thank all the patients who agreed to participate in the clinical trials; the members of the Kidney Transplant Programs, HLA Laboratory, Transplant Immunology Laboratory, and Renal Pathology at Cedars-Sinai Medical Center, Uppsala University, and Karolinska Institutet for efforts on behalf of the patients; and research nurses Mia Elofsson, Maria Svenaeus Lundgren, Malin Dackborn, Åsa Aringskog, and Anna Högvall and patient coordinator Ingrid Skarp for professional help. We acknowledge the enduring legacy of Paul Terasaki, who pioneered the antibody theory of transplantation. 
APPENDIX

The authors' full names and academic degrees are as follows: Stanley C. Jordan, M.D., Tomas Lorant, M.D., Jua Choi, Pharm.D., Christian Kjellman, Ph.D., Lena Winstedt, Ph.D., Mats Bengtsson, M.D., Xiaohai Zhang, Ph.D., Torsten Eich, M.D., Mieko Toyoda, Ph.D., Britt-Marie Eriksson, M.D., Shili Ge, Ph.D., Alice Peng, M.D., Sofia Järnum, Ph.D., Kathryn J. Wood, D.Phil., Torbjorn Lundgren, M.D., Lars Wennberg, M.D., Lars Bäckman, M.D., Erik Larsson, M.D., Rafael Villicana, M.D., Joe Kahwaji, M.D., Sabrina Louie, M.P.H., Alexis Kang, B.S., Mark Haas, M.D., Ph.D., Cynthia Nast, M.D., Ashley Vo, Pharm.D., and Gunnar Tufveson, M.D.

The authors' affiliations are as follows: the Comprehensive Transplant Center (S.C.J., J.C., M.T., S.G., A.P., R.V., J.K., S.L., A.K., A.V.), Transplant Immunotherapy Program (S.C.J., J.C., A.P., R.V., J.K., S.L., A.K., A.V.), Transplant Immunology Laboratory (S.C.J., M.T., S.G.), HLA Laboratory (X.Z.), and the Department of Pathology (M.H., C.N.), Cedars-Sinai Medical Center, Los Angeles; the Section of Transplantation Surgery, Department of Surgical Sciences (T. Lorant, L.B., G.T.), the Section of Molecular and Morphological Pathology (M.B., E.L.) and the Section of Clinical Immunology (T.E.), Department of Immunology, Genetics, and Pathology, and the Section of Infectious Diseases, Department of Medical Sciences (B.-M.E.), Uppsala University, Uppsala, Hansa Medical, Lund (C.K., L. Winstedt, S.J.), and the Division of Transplantation Surgery, Department of Clinical Sciences, Intervention, and Technology, Karolinska Institutet, and the Department of Transplantation Surgery, Karolinska University Hospital, Stockholm (T. Lundgren, L. Wennberg) all in Sweden; and the Nuffield Department of Surgical Sciences, Oxford University, Oxford, United Kingdom (K.J.W.).

\section{REFERENCES}

1. Evans RW, Manninen DL, Garrison LP Jr, et al. The quality of life of patients with end-stage renal disease. $\mathrm{N}$ Engl J Med 1985;312:553-9.

2. Port FK, Wolfe RA, Mauger EA, Berling DP, Jiang K. Comparison of survival probabilities for dialysis patients vs cadaveric renal transplant recipients. JAMA 1993;270:1339-43.

3. U.S. Renal Data System. USRDS 2013 annual data report: atlas of chronic kidney disease and end-stage renal disease in the United States (https://www.usrds.org/ atlas13.aspx).

4. Cecka JM, Kucheryavaya AY, Reinsmoen NL, Leffell MS. Calculated PRA: initial results show benefits for sensitized patients and a reduction in positive cross matches. Am J Transplant 2011;11:719-24. 5. Vo AA, Lukovsky M, Toyoda M, et al. Rituximab and intravenous immune globulin for desensitization during renal transplantation. N Engl J Med 2008;359: 242-51.

6. Jordan SC, Tyan D, Stablein D, et al. Evaluation of intravenous immunoglobulin as an agent to lower allosensitization and improve transplantation in highly sensitized adult patients with end-stage renal disease: report of the NIH IG02 trial. J Am Soc Nephrol 2004;15:3256-62.

7. Marfo K, Lu A, Ling M, Akalin E. Desensitization protocols and their outcome. Clin J Am Soc Nephrol 2011;6:922 36.

8. Montgomery RA, Lonze BE, King KE, et al. Desensitization in HLA-incompatible kidney recipients and survival. $\mathrm{N}$ Engl J Med 2011;365:318-26.

9. Orandi $\mathrm{BJ}$, Luo $\mathrm{X}$, Massie $\mathrm{AB}$, et al. Survival benefit with kidney transplants from HLA-incompatible live donors. N Engl J Med 2016;374:940-50.

10. Rostaing LP, Malvezzi P. HLA-incompatible kidney transplantation - worth the risk? N Engl J Med 2016;374:982-4.

11. Lefaucheur C, Loupy A, Hill GS, et al. Preexisting donor-specific HLA antibod- ies predict outcome in kidney transplantation. J Am Soc Nephrol 2010;21:1398406.

12. Jordan SC, Reinsmoen N, Peng A, et al. Advances in diagnosing and managing antibody-mediated rejection. Pediatr $\mathrm{Ne}$ phrol 2010;25:2035-45.

13. Vo AA, Petrozzino J, Yeung K, et al. Efficacy, outcomes, and cost-effectiveness of desensitization using IVIG and rituximab. Transplantation 2013;95:8528.

14. Archdeacon P, Chan M, Neuland C, et al. Summary of FDA antibody-mediated rejection workshop. Am J Transplant 2011;11:896-906.

15. Brezski RJ, Vafa O, Petrone D, et al. Tumor-associated and microbial proteases compromise host IgG effector functions by a single cleavage proximal to the hinge. Proc Natl Acad Sci U S A 2009;106: 17864-9.

16. von Pawel-Rammingen U, Björck L. IdeS and SpeB: immunoglobulin-degrading cysteine proteinases of Streptococcus pyogenes. Curr Opin Microbiol 2003;6:505 .

17. Reinsmoen $\mathrm{NL}$, Lai $\mathrm{CH}$, Vo $\mathrm{A}$, et al. Acceptable donor-specific antibody levels allowing for successful deceased and living donor kidney transplantation after desensitization therapy. Transplantation 2008;86:820-5.

18. Haas M, Sis B, Racusen LC, et al. Banff 2013 meeting report: inclusion of C4d-negative antibody-mediated rejection and antibody-associated arterial lesions. Am J Transplant 2014;14:272-83.

19. Winstedt L, Järnum S, Nordahl EA, et al. Complete removal of extracellular IgG antibodies in a randomized dose-escalation phase I study with the bacterial enzyme IdeS - a novel therapeutic opportunity. PLoS One 2015;10(7):e0132011.

20. Loupy A, Lefaucheur C, Vernerey D, et al. Complement-binding anti-HLA antibodies and kidney-allograft survival. N Engl J Med 2013;369:1215-26.
21. Toyoda M, Shin BH, Ge S, et al. Impact of desensitization on antiviral immunity in HLA-sensitized kidney transplant recipients. J Immunol Res 2017;2017: 5672523.

22. Vo AA, Choi J, Cisneros K, et al. Benefits of rituximab combined with intravenous immunoglobulin for desensitization in kidney transplant recipients. Transplantation 2014;98:312-9.

23. Zachary AA, Lucas DP, Montgomery RA, Leffell MS. Rituximab prevents an anamnestic response in patients with cryptic sensitization to HLA. Transplantation 2013;95:701-4.

24. Patel R, Terasaki PI. Significance of the positive crossmatch test in kidney transplantation. N Engl J Med 1969;280: 735-9.

25. Jeannet M, Pinn VW, Flax MH, Winn HJ, Russell PS. Humoral antibodies in renal allotransplantation in man. $\mathrm{N}$ Engl J Med 1970;282:111-7.

26. Loupy A, Jordan SC. Transplantation: donor-specific HLA antibodies and renal allograft failure. Nat Rev Nephrol 2013;9: 130-1.

27. von Pawel-Rammingen U, Johansson BP, Björck L. IdeS, a novel streptococcal cysteine proteinase with unique specificity for immunoglobulin G. EMBO J 2002; 21:1607-15.

28. Carapetis JR, Steer AC, Mulholland EK, Weber M. The global burden of group A streptococcal diseases. Lancet Infect Dis 2005;5:685-94.

29. Järnum S, Bockermann R, Runström A, Winstedt L, Kjellman C. The bacterial enzyme IdeS cleaves the IgG-type of B cell receptor (BCR), abolishes BCR-mediated cell signaling, and inhibits memory B cell activation. J Immunol 2015;195: 5592-601.

30. Takahashi R, Yuki N. Streptococcal IdeS: therapeutic potential for GuillainBarré syndrome. Sci Rep 2015;5:10809.

Copyright (c) 2017 Massachusetts Medical Society. 